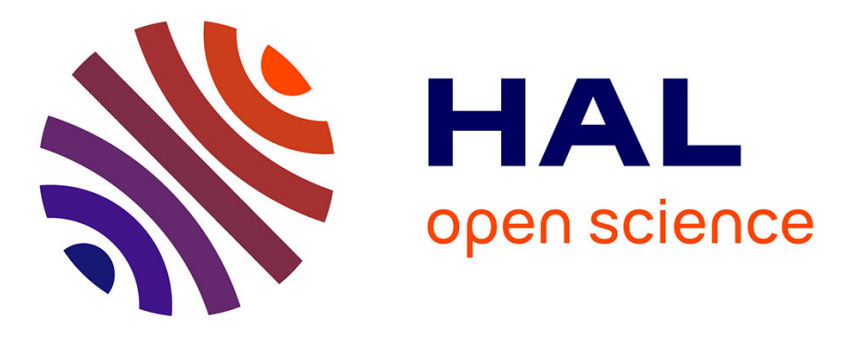

\title{
Cells Detection Using Segmentation Competition
}

Emmanuelle Poulain, Sylvain Prigent, Emmanuel Soubies, Xavier Descombes

\section{To cite this version:}

Emmanuelle Poulain, Sylvain Prigent, Emmanuel Soubies, Xavier Descombes. Cells Detection Using Segmentation Competition. ISBI - International Symposium on Biomedical Imaging, Apr 2015, Brooklyn, United States. 10.1109/ISBI.2015.7164090 . hal-01113167

\section{HAL Id: hal-01113167 \\ https://hal.inria.fr/hal-01113167}

Submitted on 4 Feb 2015

HAL is a multi-disciplinary open access archive for the deposit and dissemination of scientific research documents, whether they are published or not. The documents may come from teaching and research institutions in France or abroad, or from public or private research centers.
L'archive ouverte pluridisciplinaire $\mathbf{H A L}$, est destinée au dépôt et à la diffusion de documents scientifiques de niveau recherche, publiés ou non, émanant des établissements d'enseignement et de recherche français ou étrangers, des laboratoires publics ou privés. 


\title{
CELLS DETECTION USING SEGMENTATION COMPETITION
}

\author{
Emmanuelle Poulain ${ }^{1}$, Sylvain Prigent ${ }^{1}$, Emmanuel Soubies ${ }^{2}$, Xavier Descombes ${ }^{1 *}$ \\ ${ }^{1}$ INRIA, 2003 route des Lucioles, BP93, 06902 Sophia Antipolis Cedex, France \\ ${ }^{2}$ Université Nice Sophia Antipolis, I3S, CNRS, Sophia Antipolis, France
}

\begin{abstract}
In this paper we address the problem of cells detection from microscopy images. We construct a dictionary of candidate shapes obtained from previous segmentation maps and define an energy function to select the best candidates. The energy minimization is performed by an iterative graph cut algorithm. The proposed approach optimally combines the segmentation maps obtained with different methods and/or parameters. We show on synthetic and real data that this process allows to drastically improve the performance of each individual segmentation.
\end{abstract}

Index Terms - Cells detection, Segmentation, Graph Cut

\section{INTRODUCTION}

Image segmentation has been widely investigated in particular in the context of bioimaging for cells detection. In some cases, the background is clearly identifiable so that a binary mask of the objects can be computed using simple techniques such as thresholding. Therefore, isolated objects are easily recognizable while splitting clusters of objects, which are connected components in the binary mask, remains a challenging task. In fluorescent microscopy devices used for live imaging - e.g. confocal, biphoton, Selective Plane Illumination Microscope (SPIM) - an additional difficulty comes from the multiple degradations of the acquired images such as strong noise, spatially varying blur and light attenuation which makes the segmentation a hard task even for selecting a suitable threshold for the background. Since many years, researchers have developed several methods to perform such segmentation. An efficient approach consists in generating seeds that define regions using geometric information through a distance, as in the markers controlled watershed algorithm [1], or image gradient for the active contour approach [2]. The accuracy of these methods is strongly dependent to the seed generation. Bayesian approaches, such as marked point process, avoid this bottleneck by selecting randomly generated shapes through the minimization of an energy function [3]. However, they are restricted to low dimensional parametric shapes, such as disks or ellipses, due to computational issues. Tuning the parameters of the segmentation algorithms mentioned above in order to obtain accurate results on the whole image can also be extremely tricky whereas it is much easier to obtain accurate results on different parts of the image using different sets of parameters. To overcome these limits we propose to combine both approaches by generating shapes from state of the art segmentation algorithms using random seeds and/or different sets of parameters. These shapes will define a dictionary of candidates from which a competition process, using the Multiple Birth and Cut algorithm [4], will then extracts the most relevant shapes. The proposed method differs from the recent multiple over-segmentations

\footnotetext{
${ }^{*}$ All authors are within the MORPHEME team (INRIA/I3S/iBV) Contact: xavier.descombes@inria.fr
}

fusion algorithms [5, 6] (and reference therein) in the sense that our method consider objects with shape prior and interactions between them. The main idea in $[5,6]$ is that "correct" boundaries are present in each over-segmentation which is not the case for spurious edges. Then they aim to jointly merge segments of the different over-segmentations. Unlike these methods, our approach extracts objects from different segmentation maps and selects the most relevant introducing data fidelity and interaction energies. By this way, we are able to split clusters of objects that is an important issue in cells detection.

The paper is organized as follows. Section 2 describes the selection approach and Section 3 presents numerical results on synthetic data and on a multicellular tumor spheroide slice. We use two different state of the art segmentation methods to build the dictionary of shapes and compare the performance of our competition approach with the ImageJ particle analyser.

\section{BEST SHAPES SELECTION FROM A DICTIONARY}

Let $\mathcal{D}$ be a dictionary of candidate shapes defined from different segmentation maps. The main idea of this work is to propose a method to select the most relevant atoms of this dictionary in order to improve each individual segmentation. It is important to note that the dictionary can be constructed with any segmentation algorithms by extracting the connected components of the resulting maps. Then the method presented in the latter can be viewed as a process mixing different segmentation maps to provide the "best" combination taking the advantages of each individual ones.

We consider the set $\mathcal{O}=\mathcal{P}(\mathcal{D})$ of all subsets of $\mathcal{D}$. Let us define a pairwise interaction energy function on this configuration space as follows: $\forall o=\left\{o_{1}, \ldots, o_{i}, \ldots, o_{m}\right\} \in \mathcal{O}$,

$$
U(o)=\sum_{i=1}^{m} U_{1}\left(o_{i}\right)+\sum_{\substack{i, j=1 \\ o_{i} \sim o_{j}}}^{m} U_{2}\left(o_{i}, o_{j}\right),
$$

where $U_{1}$ and $U_{2}$ are respectively data fidelity and prior energies and $\sim$ denotes an interaction relationship between two objects of $o \in \mathcal{O}$. The most relevant shapes are selected by minimizing this energy function over all the subsets of objects $o \in \mathcal{O}$.

Without loss of generality but for simplicity, we define energies in the $2 \mathrm{D}$ case. In this paper we consider a binary prior that allows controlling the maximum overlap between objects as follows: $\forall\{i, j\} \in\{1, \cdots, m\}^{2}$,

$$
U_{2}\left(o_{i}, o_{j}\right)=\left\{\begin{array}{lll}
0 & \text { if } & \frac{\left|o_{i} \cap o_{j}\right|}{\min \left(\left|o_{i}\right|,\left|o_{j}\right|\right)} \leq T_{o} \\
\infty & \text { if } & \frac{\left|o_{i} \cap o_{j}\right|}{\min \left(\left|o_{i}\right|,\left|o_{j}\right|\right)}>T_{o}
\end{array}\right.
$$

where $|u|$ represents the area of object $u$ and $T_{o}$ is the maximum accepted percentage of overlap between two objects. 
The first order term $U_{1}$ provides some constraints on the geometry of objects and/or on the object intensity in the image. Let $u$ be an object and $r_{i}$ (resp. $r_{c}$ ) the radius of its inscribed (resp. circumscribed) circle. We define a term to control both the size and the shape of cells, which should not deviate too much from a disk, as follows: we consider the function $f(x)=(x-1+\lambda)(x-1-\lambda)$ that is minimum for $x=1$ and null for $x=1-\lambda$ and $x=1+\lambda$. Then $U_{1}$ is defined by:

$$
U_{1}(u)=\min \left(\frac{r_{c}}{r}, \frac{r}{r_{c}}\right)^{2} \times f\left(\frac{r_{i}}{r_{c}}\right),
$$

where $r$ is a parameter estimating the radius of a typical cell. In practice, to estimate $r$ we compute the median size of the connected components in the thresholded image and compute the radius of a disk with the same size. Using (3), the shapes that deviate too much from the disk shape (i.e. $\left|\frac{r_{i}}{r_{c}}-1\right|>\lambda$ ) are eliminated.

When cells within a cluster can be distinguished using their intensity, we consider as first order potential a distance between pixels intensities within and outside the object [3]. Let $\mu_{1}$ (resp. $\mu_{2}$ ) and $\sigma_{1}^{2}$ (resp. $\sigma_{2}^{2}$ ) denote the mean and the variance of pixel intensity inside the object (resp. in the external border of the object). We consider the following first order potential:

$$
U_{1}(u)= \begin{cases}1 & \text { if } d_{B}(u)<d_{0} \\ \exp \left(-\frac{d_{B}(u)-d_{0}}{s}\right)-1 & \text { if } d_{B}(u) \geq d_{0}\end{cases}
$$

where:

$$
d_{B}(u)=\frac{\left(\mu_{1}-\mu_{2}\right)^{2}}{4 \sqrt{\sigma_{1}^{2}+\sigma_{2}^{2}}}-\frac{1}{2} \log \frac{2 \sigma_{1} \sigma_{2}}{\sigma_{1}^{2}+\sigma_{2}^{2}},
$$

$d_{0}$ is a threshold defining the minimum allowed contrast for an object and $s$ controls the slope of $U_{1}$ for acceptable objects. With this potential, shapes with higher contrast are preferred.

To select the best set of candidates, the algorithm, described in Algorithm 1, iteratively compares the current configuration of objects with a random one and select the best subset of their union.

\section{Algorithm 1. Select Candidates}

$1 i=1$, Select randomly a set $P_{1}$ of $m_{p}$ shapes in $\mathcal{D}$ such that:

$$
\forall\left(p, p^{\prime}\right) \in P_{1}^{2}, p \neq p^{\prime} \Rightarrow \frac{\left|p \cap p^{\prime}\right|}{\min \left(|p|,\left|p^{\prime}\right|\right)} \leq T_{o} .
$$

\section{Repeat until convergence:}

2.a Select randomly a set $Q_{i}$ of $m_{q}$ shapes in $\mathcal{D}$ such that:

$$
\forall\left(q, q^{\prime}\right) \in Q_{i}^{2}, q \neq q^{\prime} \Rightarrow \frac{\left|q \cap q^{\prime}\right|}{\min \left(|q|,\left|q^{\prime}\right|\right)} \leq T_{o} .
$$

\section{2.b Compute}

$$
P_{i+1}=\underset{o \subset P_{i} \cup Q_{i}}{\arg \min } U(o),
$$

using the graph cut approach described in [4] and update $i=i+1$.

To apply the graph cut approach for solving the minimization problem (6) between two sets of shapes, we have to satisfy the regularity condition defined in [7]. As we consider binary repulsive interactions (two overlapping shapes are not compatible), the resulting cost function in (6) is nonregular. In order to make it regular, one approach (see [4]) consists in interchanging the sense of 0 and 1 in the binary graph for one set. We then consider a binary Markov random field on the graph $\mathcal{G}_{P, Q}$ with nodes in $P \cup Q$. Edges are given by the interaction $\sim$ defined in equation (2). The binary value associated with each node - denoted $n_{p}$ (resp. $n_{q}$ ) for a node associated to $p \in P$ (resp. in $q \in Q$ ) - has the following interpretation:

- $n_{p}=1$ (resp. $n_{q}=0$ ) means that $p$ (resp. $q$ ) is kept in the configuration

- $n_{p}=0$ (resp. $n_{q}=1$ ) means that $p$ (resp. $q$ ) is removed from the configuration

Consider the first order potentials as follows:

$$
\begin{aligned}
& \forall p \in P, D_{P, Q}\left(n_{p}\right)=U_{1}(p) \delta\left(n_{p}=1\right) \\
& \forall q \in Q, D_{P, Q}\left(n_{q}\right)=U_{1}(q) \delta\left(n_{q}=0\right)
\end{aligned}
$$

and the second order interaction as follows: $\forall(p, q) \in P \times Q$,

$V_{P, Q}\left(n_{p}, n_{q}\right)= \begin{cases}U_{2}(p, q)\left[\delta\left(n_{p}=1\right) \wedge \delta\left(n_{q}=0\right)\right] & \text { if } p \sim q \\ 0 & \text { otherwise }\end{cases}$

We obtain the global energy of the MRF as follows:

$$
U_{P, Q}(\mathbf{n})=\sum_{u \in P \cup Q} D_{P, Q}\left(n_{u}\right)+\sum_{\substack{\{p, q\} \in P \times Q \\ p \sim q}} V_{P, Q}\left(n_{p}, n_{q}\right)
$$

where $\mathbf{n}=\left\{n_{p}, p \in P, n_{q}, q \in Q\right\}$.

Property 1. Let $\mathbf{n}$ be the set of nodes associated to the objects in $P \cup Q$ and $m_{p}$ and $m_{q}$ be respectively the number of objects in $P$ and $Q$. Then there exists a bijection:

$$
\begin{array}{ccc}
\mathcal{B}: \mathcal{P}(P \cup Q) & \longrightarrow & \{0,1\}^{m_{p}+m_{q}} \\
o & \longmapsto & \mathbf{n}
\end{array},
$$

such that:

$$
\forall o \in \mathcal{P}(P \cup Q), U_{P, Q}(\mathcal{B}(o))=U(o),
$$

and $\hat{o}=\mathcal{B}^{-1}(\hat{\mathbf{n}})$ where:

$$
\hat{o}=\underset{o \in \mathcal{P}(P \cup Q)}{\arg \min } U(o) \quad \text { and } \quad \hat{\mathbf{n}}=\underset{\mathbf{n} \in\{0,1\}^{m_{p}+m_{q}}}{\arg \min } U_{P, Q}(\mathbf{n})
$$

Proof. By definition of $U_{P, Q}$ given in (9) it is clear that the bijection $\mathcal{B}$ defined by $\forall o \in \mathcal{P}(P \cup Q), \mathbf{n}=\mathcal{B}(o)$ where $\forall p \in P, \mathbf{n}_{p}=$ $\delta(p \in o)$ and $\forall q \in Q, \mathbf{n}_{q}=1-\delta(q \in o)$ satisfy (11). The last statement of the property is straightforward from the previous points.

Property 2. The energy $U_{P, Q}$ satisfies the regularity condition as defined in [7] that is:

$$
V_{P, Q}(1,1)+V_{P, Q}(0,0) \leq V_{P, Q}(1,0)+V_{P, Q}(0,1)
$$

Proof. The proof is straightforward from the definition (8).

From Property 2, we can compute the minimal graph cut to minimize the energy defined in (9) (see Figure 1). Then Property 1 allow us to get the optimal subset $\hat{o} \subset P \cup Q$ minimizing the energy (1). 

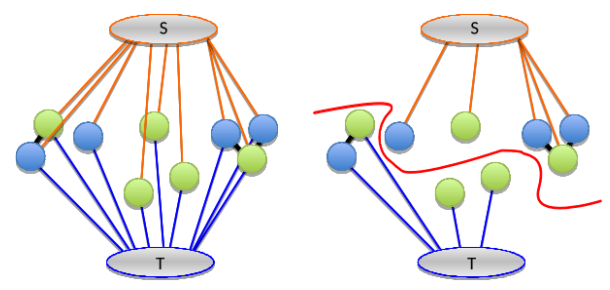

Fig. 1. Left: graph constructed from $P$ (in blue) and Q (in green) configurations. Edges are added between all nodes and $\mathrm{S}$ (source in orange) and $\mathrm{T}$ (sink in blue) as detailed in [4]. Non overlapping constraint is modeled by dark edges between green and blue nodes. Right: one possible minimal cut in red (which split the graph in two subsets, one containing the source and the other the sink, by removing edges minimizing the global weight of all removed edges). Objects corresponding to blue (resp. green) nodes linked to $\mathrm{S}$ (resp. T) are preserved. Edges between blue and green nodes have an infinite weight which prevents them being cut.

\section{NUMERICAL EXPERIMENTS}

\subsection{Candidate shapes generation}

As mentioned in the previous section, we first need to build a dictionary of candidate shapes. To achieve this goal, a large number of algorithms from the state of the art are available. In this paper we will use two standard methods which are markers controlled watershed [1] (or SKeleton by Influence Zones) and Fast Marching (FM) [8] (a curve evolution based approach). We show that even with these "basic" techniques for shape generation, our competition process improves significantly the resulting segmentation. Let's now present different ways to generate the dictionary.

Random Seed SKIZ (RS-SKIZ) is the simplest method which compute the following steps -1 . extract the skeleton of the thresholded image to provide "central lines" of each connected component, - 2. generate random seeds on this skeleton to initialize the SKIZ algorithm, -3 . intersect the obtained areas with the thresholded image, -4 . extract connected components to obtain candidate shapes. An illustration is given on Figure 2. The three last steps are repeated a fixed number of times to complete the dictionary.

Multi-Thresholds Random Seed SKIZ (MTRS-SKIZ) repeats the RS-SKIZ for different threshold levels which allows to obtain a multi-levels description in the dictionary.

Multi-Parameters Random Seed FM (MPRS-FM) performs the following steps -1 . generate random seeds on the whole binary mask, obtained by thresholding, to initialize the FM algorithm, -2 . extract connected components to obtain candidate shapes, -3 . repeat steps 1 and 2 a fixed number of times. Then these three steps are repeated using different values for the parameter used to stop the expansion. This provides multi-scale objects for the dictionary.

In all cases, the random seeds generation follows a Poisson process. The density of this Poisson process is related to the scale of the final segmentation (i.e. the size of the detected objects).

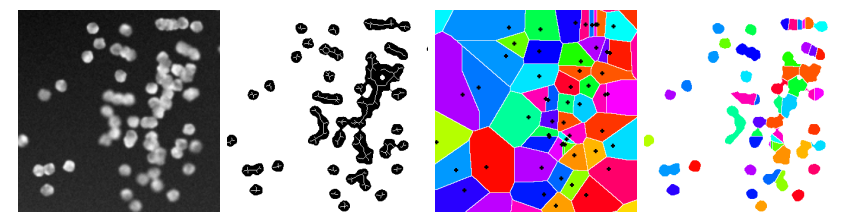

Fig. 2. Example of candidate shape generation with the four steps of RS-SKIZ method. From left to right: crop $-340 \times 340 \mathrm{px}-$ of the initial synthetic image $-950 \times 950 \mathrm{px}-$, skeleton on the thresholded image, random seeds with associated SKIZ and obtained candidates.

\subsection{Results}

We will now compare our approach with the particle analysis algorithm proposed in ImageJ. This algorithm consists of two steps. In the first step, the initial image is thresholded and a distance map is computed inside the obtained connected components. A SKIZ is then computed where the seeds are given by the local maxima of the distance map. In the second step, the obtained region are selected using their size and a circularity parameter. This can be considered as the standard approach when using the markers controlled watershed principle or the "best" segmentation which can be obtained by applying once the four steps of RS-SKIZ.

We first consider a synthetic image of cells partially given on Figure 2 (left) and for which we have the ground truth ${ }^{1}$. In our competition approach, we decide here to generate the dictionary using the RS-SKIZ presented above. The threshold value is determined with the Otsu approach. In this image cells have homogeneous intensity and similar shapes. Therefore, we consider the geometric first order potential defined by (3) in the competition process described in Algorithm 1. Table 1 summarizes the results obtained with the ImageJ particle analyzer, the SKIZ on one realization of the Poisson process (i.e. the segmentation obtained by applying one time the four steps of RS-SKIZ) and the proposed approach. The particle analyzer algorithm provides a much better result than those obtained by randomly generated seeds for the SKIZ. However, the proposed selection approach from the randomly generated shape dictionary clearly outperforms this algorithm. Note that the number of false alarm is lower for PA1 and the prec is the highest for this method but this is not significant since the corresponding number of nuclei in the resulting segmentation is highly under the number of nuclei in the ground truth. Figure 3 shows the results obtained by PA2 (left) and by the proposed approach (right). We can notice the better splitting of cell clusters with the latter.

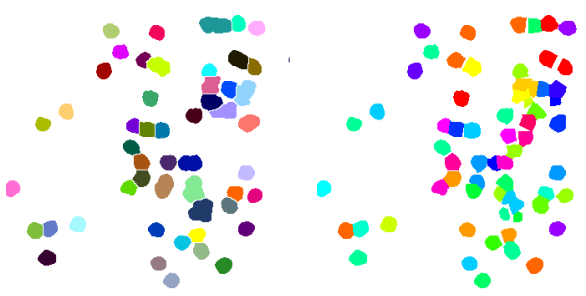

Fig. 3. Results obtained with PA2 (left) and SC500 (right) on the image of the Figure 2 (top-left). These results correspond to a part of the image used for Table 1.

\footnotetext{
${ }^{1}$ See http://www.cellimagelibrary.org/
} 


\begin{tabular}{|c|c|c|c|c|c|c|c|}
\hline & M & N & CD & MD & FA & prec & rec \\
\hline W100 & 279 & 175 & 153 & 126 & 22 & 0.874 & 0.548 \\
\hline B100 & 279 & 264 & 201 & 78 & 77 & 0.708 & 0.720 \\
\hline SC100 & 279 & 274 & 256 & 23 & 18 & 0.934 & 0.918 \\
\hline SC500 & 279 & $\mathbf{2 7 6}$ & $\mathbf{2 6 2}$ & $\mathbf{1 7}$ & 16 & 0.942 & $\mathbf{0 . 9 3 9}$ \\
\hline PA1 & 279 & 80 & 78 & 201 & $\mathbf{2}$ & $\mathbf{0 . 9 7 5}$ & 0.280 \\
\hline PA2 & 279 & 229 & 194 & 85 & 35 & 0.847 & 0.695 \\
\hline
\end{tabular}

Table 1. Results obtained with the ImageJ Particle Analyzer for two different parameter values (PA1 and PA2), with one realization of random seeds (W100 :mean value on 100 samples and B100: best result over 100 samples) and with the proposed Segmentation Competition approach (SC100 for 100 realizations of random seeds and SC500 for 500). The dictionary generation is performed according to RS-SKIZ with a threshold value computed using Otsu approach. The columns represent: $\mathrm{M}=$ number of nuclei in the ground truth, $\mathrm{N}$ $=$ number of nuclei in the segmentation output, $\mathrm{CD}=$ correct detection (at least $60 \%$ of each object overlap with the ground truth), MD $=$ missed detection, $\mathrm{FA}=$ false alarms, $\operatorname{prec}=\frac{C D}{N}, \mathrm{rec}=\frac{C D}{M}$.

Finally, we consider a 2D slice of a multicellular tumor spheroid acquired using a SPIM microscope presented on Figure 4 (top line left). In this case, the cells exhibit some size variability as well as intensity heterogeneity. Thus, we consider the first order potential defined by equation (4). On Figure 4 we present the result of the ImageJ particle analyzer (top line right) and the ones produced by our approach considering the different methods presented in the previous section for the generation step; RS-SKIZ (middle line left), MTRSSKIZ (middle line right), MPRS-FM (bottom line left) and a combination of the MTRS-SKIZ and MPRS-FM - i.e. we concatenate the dictionaries of shapes obtained with both methods - (bottom line right). A brief description of used parameters is given in the legend of the Figure 4. All the results obtained with the proposed segmentation competition approach outperform the particle analyzer result which undersegments the top right part of the image. Moreover, we can observe that edges are better delineated with the use of MTRSSKIZ for generating the dictionary and that is improved with shapes obtained using MPRS-FM. This is due to the watershed procedure on a distance map used in the SKIZ algorithm which do not consider the image gradient as in the Fast Marching algorithm. Finally the result is again improved by combining the dictionaries of shapes obtained with MTRS-SKIZ and MPRS-FM. Despite various sort of degradation on this image (strong blur, noise, stripes), the variability of objects size, the intensity heterogeneity and the presence of clusters of objects, we obtain an accurate segmentation. This show the robustness of the proposed approach.

\section{CONCLUSION}

In this paper we have proposed a segmentation selection approach. Using graphcut we select the most relevant shapes from a dictionary constructed by previous segmentation maps. We have considered markers controlled watershed and Fast Marching algorithm initialized with randomly generated seeds and different sets of algorithm parameters to generate the candidate shapes. The results show that the final segmentation outperforms individual ones and the particle analyzer that provides the "best" segmentation obtained from a single watershed. Results on a high degraded real image have shown the robustness of the method. This selection procedure can be applied on segmentation maps obtained from more sophisticated methods. Currently the selection model is based on a geometric prior and a non overlapping constraint. In the future, we plan to

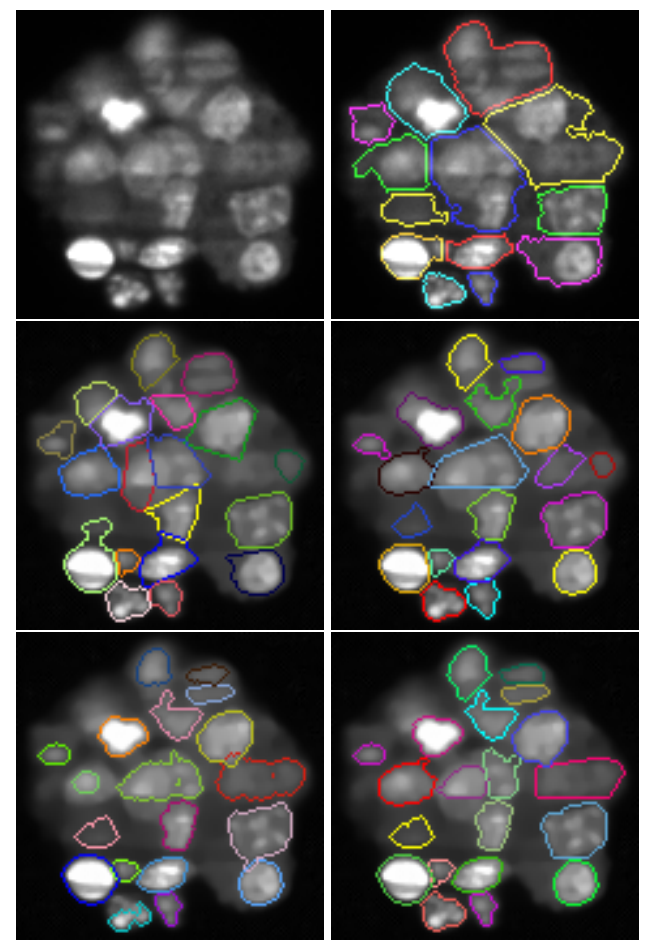

Fig. 4. Spheroids of tumor cells stained with a fluorescent nuclear marker (top line left). Fiji Particle Analyzer result (top line right). Results of our approach with dictionary generated by RS-SKIZ with 800 repetitions and a threshold value fixed to 70 (middle line left) MTRS-SKIZ with 80 repetitions and 10 different thresholds (middle line right) MPRS-FM with 100 repetitions and 8 different values for the Fast Marching parameter used to stop the expansion (bottom line left), concatenation of MTRS-SKIZ and MPRS-FM dictionaries (bottom line right).

add attractions between regions that necessitates to revisit the graph construction.

Acknowledgment: This work was supported by the French Government (National Research Agency) through the "Investments for the Future" LABEX SIGNALIFE : program reference ANR-11LABX-0028-01. The authors would like to thank Valerie Lobjois and ITAV imaging facility for providing the spheroid image.

\section{REFERENCES}

[1] P. Soille, Morphological image analysis: principles and applications, SpringerVerlag New York, Inc., 2003.

[2] S. Pop, et al "A fast and automated framework for extraction of nuclei from cluttered 3d images in fluorescence microscopy," in ISBI. IEEE, 2011.

[3] X. Descombes, Stochastic geometry for image analysis, John Wiley \& Sons, 2011.

[4] A. Gamal Eldin et al "Multiple birth and cut algorithm for multiple object detection," Journal of Multimedia Processing and Technologies, 2012.

[5] Diana L Delibaltov et al "A linear program formulation for the segmentation of ciona membrane volumes," in MICCAI, Springer, 2013.

[6] Daniel Glasner et al "Contour-based joint clustering of multiple segmentations," in $C V P R$. IEEE, 2011.

[7] Y. Boykov et al "Fast approximate energy minimization via graph cuts," PAMI, vol. 23 , no. 11,2001

[8] J.A. Sethian, Level set methods and fast marching methods: evolving interfaces in computational geometry, fluid mechanics, computer vision, and materials science, vol. 3, Cambridge university press, 1999. 